\title{
Myoelectric activity of the muscular layer of the abdominal aorta in pigs in vivo
}

\author{
Albert Czerski ${ }^{1}$, Jan Gnus ${ }^{2}$, Willy Hauzer ${ }^{2}$, Kornel Ratajczak ${ }^{3}$, Wojciech Zawadzki ${ }^{1}$, \\ Wojciech Witkiewicz ${ }^{2}$, Stanisław Ferenc ${ }^{2}$, Agnieszka Rusiecka ${ }^{1}$, Maciej Janeczek ${ }^{1}$ \\ ${ }^{1}$ Wroclaw University of Environmental and Life Sciences, Institute of Animal Physiology, \\ Department of Biostructure and Animal Physiology, Wrocław, Poland \\ ${ }^{2}$ Research and Development Center in Wroclaw, Provincial Specialized Hospital, \\ General and Vascular Surgery Ward, Wrocław, Poland \\ ${ }^{3}$ Wroclaw University of Environmental and Life Sciences, Department of Clinical Surgery, Wrocław, Poland
}

Received October 19, 2011

Accepted May 16, 2012

\begin{abstract}
The study was conducted on 5 pigs weighing 20-30 kg. Bipolar electrodes were surgically implanted into the muscular layer of the vascular smooth muscle cell of the abdominal aorta just below the renal arteries. Myoelectric activity characterized by the appearance of changes of membrane potential was found. Changes of potential could be divided according to the amplitude, duration and frequency into first-, second- and third-order waves. First-order waves appeared with a mean frequency of $128 \pm 14 / \mathrm{min}$. The mean wave amplitude was $0.150 \pm 0.03 \mathrm{mV}$, and the mean duration was $0.43 \pm 0.05 \mathrm{~s}$. They were closely correlated with the electrocardiogram and blood pressure changes. Second-order waves appeared with a mean frequency of $15.9 \pm 4.4 / \mathrm{min}$. They were characterized by a mean duration of $2.69 \pm 1.5 \mathrm{~s}$. The mean amplitude of the discharge was $0.205 \pm 0.157 \mathrm{mV}$ for the second-order wave, they were correlated with the animal's respiratory action. Third-order waves appeared with a mean frequency of $4.03 \pm 1.07 / \mathrm{min}$. They were characterized by a mean duration of $11.81 \pm 5.3 \mathrm{~s}$. The mean amplitude of the discharge was $0.345 \pm 0.232 \mathrm{mV}$ for the third-order wave and they were associated with the autonomic control of the lumen of the blood vessel. For the first time the usefulness of the electromyography method in monitoring changes in the vascular smooth muscle cell of pig abdominal aorta was proved.
\end{abstract}

\section{Electromyography, membrane potential, VSMC, contractility}

The function of the abdominal aorta is to transport blood to the smaller arterial vessels under high pressure. It performs this function due to the adaptation of the anatomy and biophysical properties of the vessel wall. Generally, the wall of blood vessels is composed of an endothelial layer, a middle layer containing numerous smooth muscular coat cells with collagen and elastic fibres, and adventitia surrounding the vessel. The biophysical properties of blood vessels are determined by the mutual quantity ratio of these three elements together and the ratio of the vessel wall thickness to the internal radius. The wall of larger arteries is characterized by a low ratio of wall thickness to the internal radius (on average $1: 6$ ), (Harder et al. 1981). It is dominated by elastic fibres that provide it with considerable tensibility. The presence of smooth muscular tissue in the arteries provides control of the vessel lumen. Muscular coat contraction causes reduction in the crosssection of the vessel which results in increased vascular resistance and blood pressure. The control of the vessel lumen takes place through nerves by the autonomic nervous system and hormonally by the secretion of vasoconstrictor (Guyenet 2006; Grassi et al. 2008).

The aim of this research was to study the bioelectric properties of the smooth muscular coat of the aorta in pigs and to interpret the obtained patterns, considering the fact that there is a strong correlation between bioelectric activity and contractility. This work is an introduction to the studies of the role of the muscular coat of the aorta in the mechanisms of

Address for correspondence:

Albert Czerski

Department of Biostructure and Animal Physiology

Institute of Animal Physiology

Wroclaw University of Environmental and Life Sciences

Ul. Norwida 31, 50-375 Wrocław, Poland

Phone: +48713205401

Fax: +48 71320540

E-mail: alb5@tlen.p

http://actavet.vfu.cz/ 
blood pressure regulation, adaptation of the study methods to the monitoring of contractility of the aorta, and their use in research on abdominal aortic aneurysm formation.

\section{Materials and Methods}

The experiments were conducted on five piglets weighing $20-30 \mathrm{~kg}$. The studies were performed following approval from the local $2^{\text {nd }}$ Bioethics Committee, approval no. 89/2010. In three animals, unipolar epicardial electrodes (Medtronic Inc., Minneapolis, MN 55432 USA, Cat.No. 6494) used for temporary pacemaking were surgically implanted in the muscular coat of the abdominal aorta of pigs. The procedure was performed under general anaesthesia. Laparotomy was performed from a lateral incision on the left side of the animal about 25 $\mathrm{cm}$ below the transverse processes of the lumbar spine. Access to the aorta was obtained without peritoneotomy. After slight uncovering of the abdominal aorta, the electrodes were implanted. In total, six electrodes ( 3 pairs of 2 electrodes) were used. The electrodes were implanted below the renal arteries in the wall of the abdominal aorta at a distance of approximately $2.5-3 \mathrm{~cm}$. between the pairs of electrodes. The distance between the electrodes in the pair was 3-4 mm, so that together they accounted for a bipolar electrode. The signal from the implanted electrodes was transmitted through cables led out on the right side of the animal around the spinous processes of the lumbar spine.

The recording of myoelectric activity of the muscular layer of the aorta was carried out in three pigs using PowerLab/ 800 (ADInstruments) connected to a computer. The Chart V.4.1 (ADInstruments) software was used for recording, playback and the analysis of the data.

In the other two pigs, myoelectric activity, changes in blood pressure in the aorta, body temperature and physical activity implants were recorded by DSI implant (Data Science International- Cat. No. TL11M3-D70CCTP). The pressure-measuring catheter was located in the aorta lumen at the height of the electrodes recording myoelectric activity changes. The signal from the implant was transferred to the recording device via telemetry. Additionally, a catheter which records changes in blood pressure using the invasive method was placed in the aorta lumen. Dataquest ART Version 4.3 Silver software was used for data collection and analysis.

After a week of convalescence, EMG (electromyography) of the wall of the abdominal aorta was performed, with simultaneous ECG (electrocardiography), registration of the respiratory rate of the animal, and changes in blood pressure using the invasive method. In experiments, the patterns of myoelectric activity of the muscle layers of the abdominal aorta in the pigs were established. The recording was performed for an hour a day using the PowerLab800 apparatus (30 records) or continuously for a week with the Data Science International apparatus.

The records of the electrical activity of the abdominal aorta were analyzed off-line. The duration, amplitude and frequency of the waves were measured. The results were presented as mean values \pm standard error $( \pm$ SEM $)$.

\section{Results}

The records of myoelectric activity of the muscular coat of the abdominal aorta was characterized by the appearance of changes of the membrane potential, which can be divided into three types of changes, conventionally called waves.

The first-order waves, which appeared with an average frequency of $128 \pm 14 \mathrm{~min}$, were distinguished in the record. The mean wave amplitude was $0.150 \pm 0.03 \mathrm{mV}$, and the mean duration was $0.43 \pm 0.05 \mathrm{~s}$. The mean heart rate calculated on the basis of ECG and the record of pressure changes in the tested animals was $132 \pm 5$ beats $/ \mathrm{min}$. Fig. 1 shows a sample record of myoelectric activity of the muscular coat of the aorta recorded with the implants from the DSI, with simultaneous registration of blood pressure changes in the aorta.

Second-order waves appeared with a mean frequency of $15.9 \pm 4.4 / \mathrm{min}$. They were characterized by mean duration of $2.69 \pm 1.5 \mathrm{~s}$. The average amplitude of the discharge was $0.205 \pm 0.157 \mathrm{mV}$ for the second-order wave. The rise of the electrical potential line (two times) due to changes caused by respiratory action (observation of the animal during the experiment) was visible. The recording was performed on the animal standing relatively still.

Third-order waves appeared with an average frequency of $4.03 \pm 1.07 / \mathrm{min}$. They were characterized by an average duration of $11.81 \pm 5.3 \mathrm{~s}$. The average amplitude of the discharge was $0.345 \pm 0.232 \mathrm{mV}$ for the third-order wave. Fig. 2 shows a recording of the myoelectric activity of the smooth muscular coat of the aorta depicting all the types of recorded waves; the third-order waves are marked. The remaining waves overlap the thirdorder waves as illustrated in the attached record. 

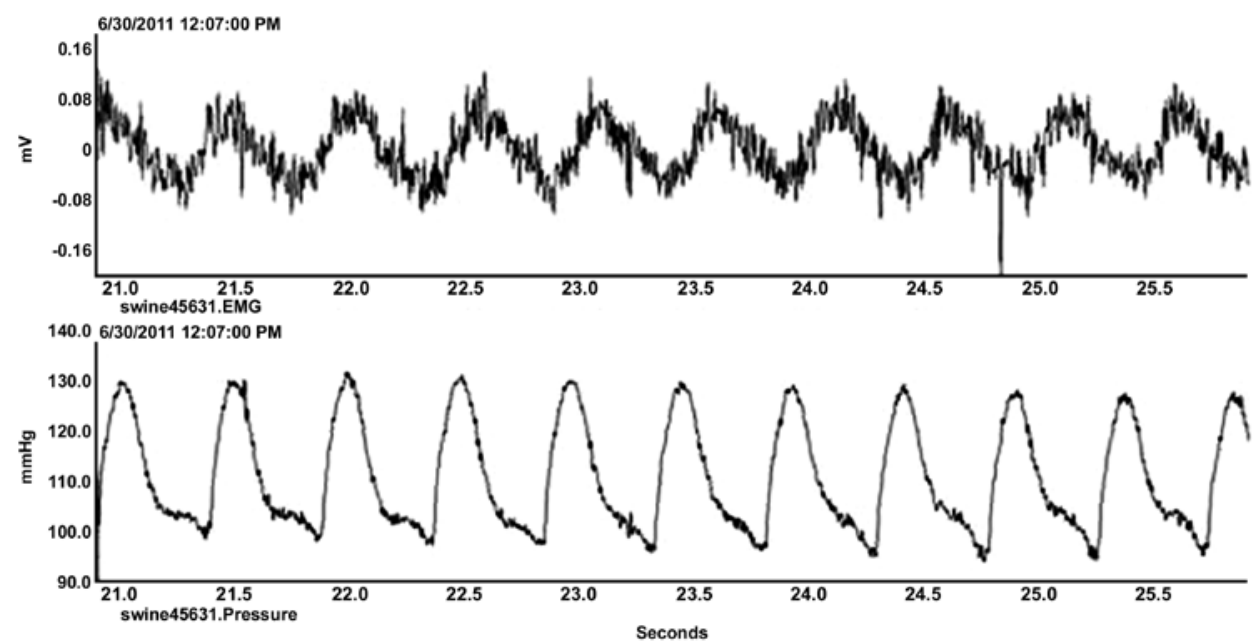

Fig. 1. Record of myoelectric activity of the muscular coat of the abdominal aorta of the pig. First-order waves are visible in the upper graph. They are closely correlated with the record of the pressure changes shown below.

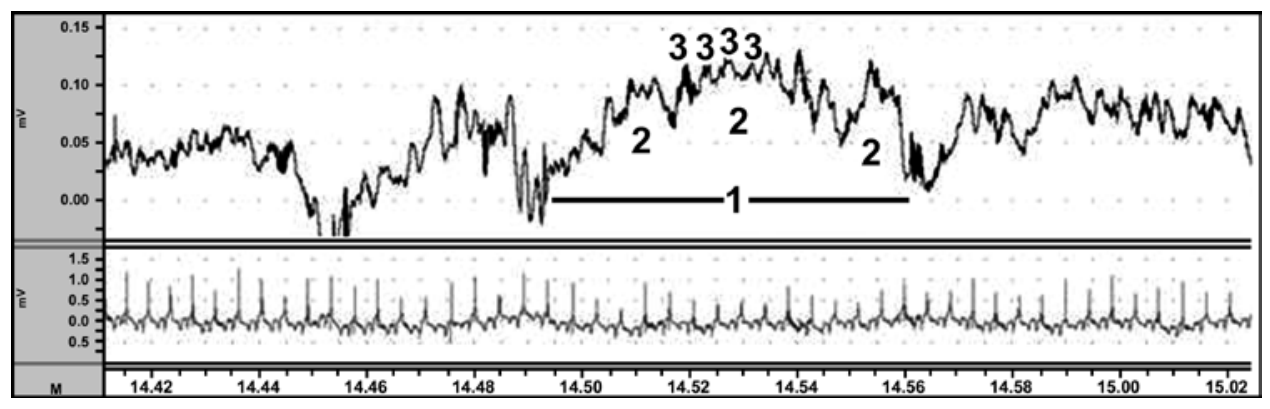

Fig. 2. Record of myoelectric activity of the abdominal aorta of the pig. In the graph, numbers $1,2,3$ are the firstorder, second-order and third-order waves, respectively. In the bottom, there is a record of the electrical activity of the heart on electrocardiogram.

\section{Discussion}

The conducted experiments demonstrated myoelectric activity of the smooth muscular coat of the abdominal aorta of the pigs by implanting bipolar electrodes used for temporary pacemaking into the wall. In the available literature, similar studies of changes in the electrical potentials in the muscle layer of the aorta were not found. Compared to the first method, the DSI implant method is less invasive for the animal, thus providing results most similar to the physiological condition. It also allows for long-term observation which may be extremely important in studies of blood pressure control as well as the etiopathogenesis of a number of lesions occurring in the blood vessels such as aneurysms, atheromatous lesions, and others. In the experiments, membrane potential fluctuations were observed, which can be divided according to frequency, duration and origin of the formation into three different types of changes, conventionally called waves. Distinguishing the different types of waves also allowed for a mathematical description of the changes in membrane potential, allowing statistical comparison of graphs and a comparison of changes in the 
patterns of muscle myoelectric activity of the muscular coat of the abdominal aorta in healthy and ill pigs in future studies.

The first-, second- and third-order waves can be isolated from the obtained records. Figs 1 and 2 show an example in high magnification of a record of myoelectric activity of the muscular coat of the abdominal aorta of the pig. The first-order waves are visible, which are closely correlated with the electrocardiogram (Fig. 2), and changes in blood pressure in the aorta measured using the invasive method (Fig. 1). This suggests that the first-order waves are formed as a result of the transfer of electrical potential along the vessel originating in the cardiac muscle, or the pulse wave affects the electrical activity of the muscle layer myocytes of the aorta generating action potentials as a result of stretching the wall of the aorta. Good implantation of the electrodes in the muscle layer of the aorta that allows reception of changes of the membrane potentials without disturbances is an important factor affecting the appearance of fist-order waves in the record. The second factor is the animal being relatively still during the recording. The best records are obtained in reclining animals or under general anaesthesia. In the recorded changes of potential, the first-order waves are very well visible and in larger fragments there are no other types of waves present in the animals put under anaesthesia by intravenous administration of xylazine and ketamine mixture. Putting the animal under anaesthesia eliminates artifacts associated with the animal's movement, lowers the heart rate and shallows respiration. Changes in the membrane potential caused by the heart beat may be associated with autonomic control of the vessel lumen. As a result of stretching of the vessel by the moving pulse wave, the muscle cells contract, preventing unduly widening of the lumen. A characteristic feature of the smooth muscular coat is its contractile response not only to electrical but also mechanical, hormonal, and chemical stimuli. A strong correlation with ECG and pulse waves unambiguously confirms the origin of the formation of the first-order wave. The role of the observed electrical changes and effects on the lumen of the vessel, which is important in the mechanisms regulating blood pressure, remains to be explicated.

The second-order waves are caused by changes in pressure in the aorta caused by the respiratory movements of the animal. Changes in pressure within the chest caused by the respiratory action affect the organs contained therein. The observed average respiration rate of the animal during the experiment ranged between $15 \pm 3 / \mathrm{min}$, depending on the motor activity of the animal. The frequency of the second-order wave and the respiratory action overlap to a large extent. The second-order waves appeared significantly less frequently in animals lying down while resting. The reason may be the marked shallowing of breath due to lack of physical activity. Most records were performed on the resting animal because that made it impossible to break the link between the apparatus and the electrodes and the resulting record lacked artifacts associated with the movement of the animal. Putting the animal under anesthesia by the administration of xylazine and ketamine also resulted in significant shallowing of the breath in the animal. There were phases of apnoea lasting tens of seconds especially after the first intravenous injection of the narcotic mixture. In the record of myoelectric activity of the muscular coat of the aorta, very weak second-order waves or even a lack of these waves were observed. Fig. 2 shows a recording of myoelectric activity of the smooth muscular coat of the aorta depicting the second-order waves. During intensified physical activity of the animal, a record of the second-order waves is often poorly readable because it is overlapped by other waves as well as the potential originated in the muscles. The second-order waves correlated with a temporary increase in blood pressure caused by deeper respiration of the animal. On the graph of pressure changes, a few second-long increases in blood pressure caused by respiratory action are observed. The changes in blood pressure caused by respiratory action can be observed on the curve of blood pressure recorded using the invasive method (Zawadzki et al. 2001). 
Similar changes are observed in the myoelectric activity of the smooth muscular coat of the aorta in the form of second-order waves appearing in the record.

The third-order waves are most probably formed as a result of fluctuations in the membrane potential induced by changes of sympathicotonia. The autonomic nervous system plays a key role in regulating blood pressure and the lumen of the vessel, which can be proved by numerous works devoted to the etiopathogenesis of arterial hypertension (Antonaccio and Kerwin 1981; K-Laflamme et al. 1997; Grassi et al. 2008). Disorders in the control of the lumen of the vessel are often caused by the malfunction of the sympathetic system, resulting in arterial hypertension. This is known as neurogenic hypertension (Guyenet 2006; Grassi et al. 2008). The neurons involved in the regulation of vascular tonus are found mainly in the spinal cord, brainstem and hypothalamus. The limbic system, cerebral cortex and midbrain structures are responsible for rapid changes in tension of the sympathetic system during different behaviors of the animal (Ros s et al. 1984; Allen 2002). Arterial blood pressure is regulated on the basis of a short- and long-term feedback control system.

The contraction of smooth muscle cells alone is associated with increased influx of calcium ions (Van Meel 1983; Asano et al. 1995; Uehata et al. 1997) into muscle cells caused by the opening of the voltage-dependent calcium channel (Pinterova et al. 2011). As a result of impulsation flowing from the sympathetic nervous system, changes in the membrane potential of muscle cells forming a vessel by a few millivolts were observed. This resulted in changes in the blood vessel diameter affecting the regulation of blood pressure (Nelson and Quayle 1995). The importance of membrane potential increase in VSMC (vascular smooth muscle cell) in the pathogenesis of hypertension has been demonstrated by Harder et al. (1981). The researchers found a relationship between the size of the membrane potential of the VSMC layer and the muscle tone. Harder et al. (1981) showed that the membrane potential of small mesenteric vessels in SHR rats (spontaneously hypertensive rat) is significantly different from that observed in normotensive WKY rats (WKY-WistarKyoto rat), but only during in vivo studies. However, in vitro no differences were observed. The observed difference in membrane potential between SHR and WKY rats in vivo can be blocked by the administration of tetrodotoxin or blockade of the $\alpha$-adrenergic receptor with phenoxybenzamine (Willems et al. 1982), which further confirms the important role of the sympathetic system in regulating the potential of VSMC.

During the recording of blood pressure using the invasive method, three types of waves induced by changes in the blood pressure can also be distinguished in the record. According to Zawadzki et al. (2001), in the model record of changes in blood pressure, we can distinguish first-, second- and third-order waves. They are induced by pressure changes under the influence of heart beat, respiratory action and changes of symphaticotonia, respectively. As demonstrated by recording VSMC myoelectric activity, the influence of the control of the vessel lumen can be studied.

Knowledge of the electrical properties of the VSMC is a key element needed to understand the processes regulating blood pressure and may also be an important indicator in the research of aortic diseases such as abdominal aortic aneurysms. The implementation of the new research method, which is the registration of myoelectric activity of VSMC, will provide valuable new data.

The results presented are an introduction to the study of the etiopathogenesis of abdominal aortic aneurysm development conducted within the framework of Wrovasc - Integrated Cardiovascular Center with the support of European Union funds.

\section{Acknowledgment}

This publication is part of the "Wrovasc - Integrated Cardiovascular Center" project, which is co-financed by the European Regional Development Fund within the Innovative Economy Operational Program, 2007-2013."European Funds - for the development of innovative economy". 


\section{References}

Allen AM 2002: Inhibition of the hypothalamic paraventricular nucleus in spontaneously hypertensive rats dramatically reduces sympathetic vasomotor tone. Hypertension 39: 275-280

Antonaccio MJ, Kerwin L 1981: Pre- and postjunctional inhibition of vascular sympathetic function by captopril in SHR. Implication of vascular angiotensin II in hypertension and antihypertensive actions of captopril. Hypertension 3: 54-62

Asano M, Nomura Y, Ito K, Uyama Y, Imaizumi Y, Watanabe M 1995: Increased function of voltage dependent $\mathrm{Ca}++$ channels and $\mathrm{Ca}++$-activated $\mathrm{K}+$ channels in resting state of femoral arteries from spontaneously hypertensive rats at prehypertensive stage. J Pharmacol Exp Ther 275: 775-783

Grassi G, Quarti-Trevano F, Dell'oro R, Mancia G 2008: Essential hypertension and the sympathetic nervous system. Neurol Sci 29: 33-36

Guyenet PG 2006: The sympathetic control of blood pressure. Nat Rev Neurosci 7: 335-346

Harder DR, Abel PW, Hermsmeyer K 1981: Membrane electrical mechanism of basilar artery constriction and pial artery dilation by norepinephrine. Circ Res 49:1237-1242

K-Laflamme A, Oster L, Cardinal R, Champlain J 1997: Effects of renin-angiotensin blockade on sympathetic reactivity and beta-adrenergic pathway in the spontaneously hypertensive rat. Hypertension 30: 278-287

Nelson MT, Quayle JM 1995: Physiological roles and properties of potassium channels in arterial smooth muscle. Am J Physiol. 268: 799-822

Pinterova M, Kunes J, Zicha J 2011: Altered neural and vascular mechanisms in hypertension. Physiol Res 60: $381-402$

Ross CA, Ruggiero DA, Park DH, Joh TH, Sved AF, Fernandez-Paradal J, Saavedra JM, Reis DJ 1984: Tonic vasomotor control by the rostral ventrolateral medulla: effect of electrical or chemical stimulation of the area containing $\mathrm{C} 1$ adrenaline neurons on arterial pressure, heart rate, and plasma catecholamines and vasopressin. J Neurosci 4: 474-494

Uehata M, Ishizaki T, Satoh H, Ono T, Kawahara T, Morishita T, Tamakawa H, Yamagami K, Inui J, Maekawa M, Narumiya S 1997: Calcium sensitization of smooth muscle mediated by a Rhoassociated protein kinase in hypertension. Nature 389: 990-994

Van Meel JC, Towart R, Kazda S, Timmermans PB, Van Zwieten PA 1983: Correlation between the inhibitory activities of calcium entry blockers on vascular smooth muscle constriction in vitro after $\mathrm{K}+$-depolarisation and in vivo after $\alpha 2$-adrenoceptor stimulation. Naunyn-Schmiedeberg's Arch Pharmacol 322: 34-37

Willems WJ, Harder DR, Contney SJ, Mccubbin JW, Stekiel WJ 1982: Sympathetic supraspinal control of venous membrane potential in spontaneous hypertension in vivo. Am J Physiol 243: 101-106

Zawadzki W, Zięba D, Dejneka J 2001: Animal Physiology. Agricultural University of Wroclaw, Wroclaw, 124 p. 